\title{
Transcatheter Atrial Septal Defect Closure in a 'Nonagenarian' with Intractable Heart Failure
}

\author{
Makiko Suzuki, Kensuke Matsumoto, Makiko Suto, Yayoi Taniguchi, Hidekazu Tanaka, \\ Hiromasa Otake, Toshiro Shinke and Ken-ichi Hirata
}

\begin{abstract}
:
A 92-year-old man was referred to our hospital with decompensated heart failure. He was treated with diuretics and inotropic agents, but the clinical response was unsatisfactory. Echocardiography incidentally revealed an atrial septal defect (ASD) with a significant left-to-right shunt and pulmonary-to-systemic-bloodflow ratio of 2.36. Because the ASD was considered to be the primary cause of his treatment-resistant heart failure, the patient underwent transcatheter ASD closure. Shortly after shunt closure, his hemodynamics significantly improved, and he was discharged nine days later. This is an extremely rare case of a nonagenarian who showed an excellent clinical course after transcatheter ASD closure.
\end{abstract}

Key words: nonagenarian, atrial septal defect, intractable heart failure, transcatheter atrial septal closure

(Intern Med 59: 1059-1063, 2020)

(DOI: 10.2169/internalmedicine.4070-19)

\section{Introduction}

Atrial septal defect (ASD) is a relatively common congenital heart disease (1). It is caused by the abnormal development of the interatrial septum during the embryonic period, which results in a partial defect of the septum. From a hemodynamic point of view, chronic blood shunting from the left to the right atrium results in chronic volume overload of the right heart and the pulmonary vasculature. However, during adolescence to young adulthood, the compliance of the right ventricle and the pulmonary vasculature is mostly sufficient to compensate for the volume overload with enlargement of the right heart and the pulmonary arterial reserve. Thus, it is relatively rare for patients with ASD to exhibit significant hemodynamic abnormalities or symptomatic manifestations during the first two to three decades of life (2). This congenital disorder often remains undiagnosed until middle age. By that time, the compliance of the left heart continuously decreases as part of the physiological aging process, resulting in a progressive increase in the left-toright shunt (3). In particular, exertion may lead to hemodynamic decompensation, with the eventual manifestation of heart failure symptoms. Thus, in most patients with ASD, transcatheter or surgical shunt closure is performed during middle age. However, ASD is occasionally diagnosed in the very elderly and the effects of treatment in these patients remain uncertain.

We herein report an extremely rare case of a nonagenarian with intractable heart failure after long-standing rightsided volume overload in an ostium secundum ASD, who was successfully treated with transcatheter ASD closure.

\section{Case Report}

The patient was a 92-year-old man who had long been treated for chronic kidney disease with renal anemia, atrial fibrillation with a slow ventricular response, and chronic heart failure of unknown cause. He was admitted to a local hospital because of progressive shortness of breath and systemic edema. He was treated with intravenous diuretics, but the response to treatment was unsatisfactory. Moreover, his renal function progressively deteriorated as a consequence of the administration of high-dose diuretics. At that point, he was transferred to our hospital for further treatment.

On admission, his blood pressure was $98 / 48 \mathrm{mmHg}$, his heart rate was 44 beats/min, and his oxygen saturation was 96\% (room air). Cardiac auscultation revealed bradycardia 

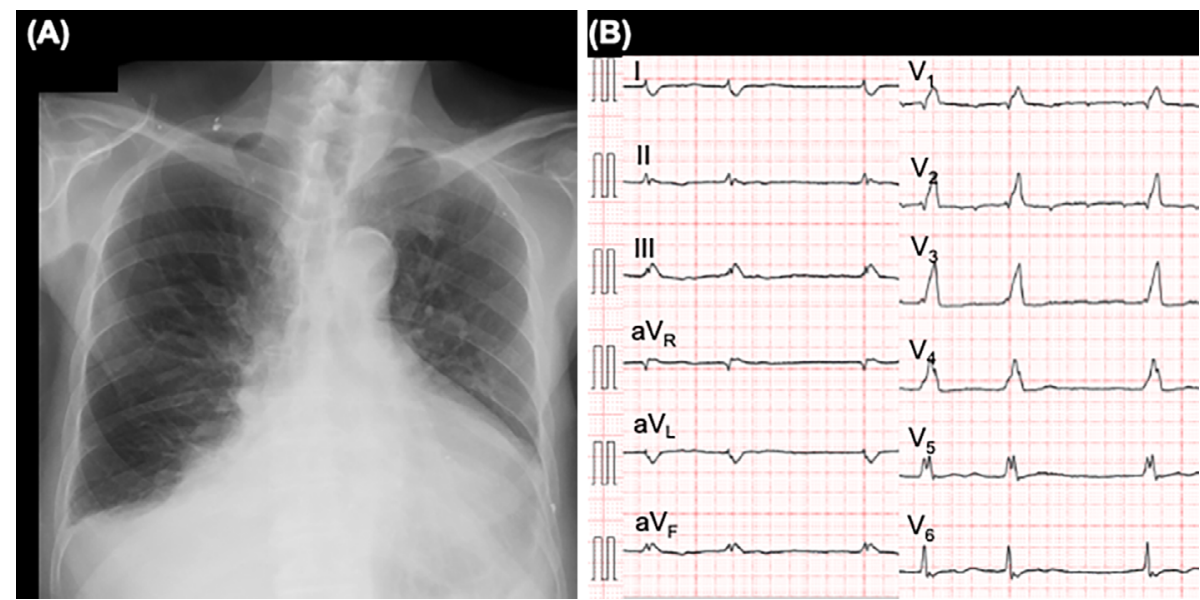

Figure 1. Radiography and electrocardiography of the 92-year-old male patient with heart failure of unknown cause. (A) A chest radiograph obtained on admission shows significant cardiomegaly and bilateral pleural effusion. (B) An electrocardiogram obtained on admission shows atrial fibrillation with a slow ventricular response.
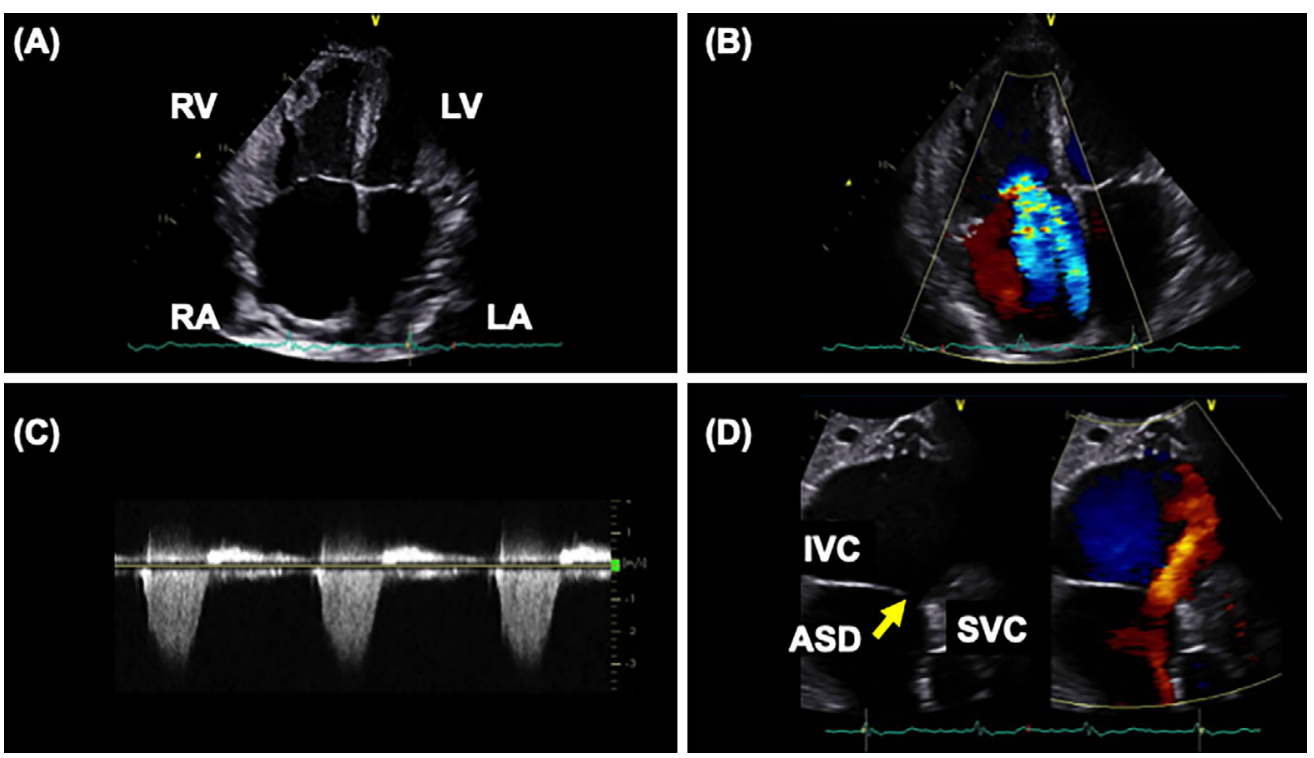

Figure 2. Transthoracic echocardiography on admission of the 92-year-old male patient with heart failure of unknown cause. (A) Both the right ventricle and atrium are significantly dilated with concomitant tricuspid annular dilatation. (B) Severe tricuspid regurgitation is visible. (C) The pressure gradient across the tricuspid value was $45 \mathrm{mmHg}$, indicating pulmonary arterial hypertension. (D) Subxyphoid view: The ostium secundum atrial septal defect (yellow arrow) was clearly visible. SVC: superior vena cava, IVC: inferior vena cava, RA: right atrium, RV: right ventricle, LA: left atrium, LV: left ventricle, ASD: atrial septal defect

with an irregular rhythm, a prominent second heart sound with fixed splitting, and a Levine III/VI systolic regurgitant murmur in the fourth intercostal space. On inspection, he had severe leg edema extending from the foot to thigh level, prominent jugular venous distention, and abdominal distention, which was consistent with right heart failure. Chest radiography showed enlargement of the cardiac silhouette and bilateral pulmonary effusion. Electrocardiography demonstrated complete right bundle branch block and atrial fibrillation with a slow ventricular response (Fig. 1). Transthoracic echocardiography revealed significant dilatation of the right atrium and ventricle (Fig. 2A and Supplementary material 1), which resulted in dilatation of the tricuspid annulus and severe tricuspid regurgitation (Fig. 2B and Supplementary material 2). Furthermore, distention of the inferior vena cava to $30 \mathrm{~mm}$ in diameter was observed without a visible respiratory change. Doppler echocardiography revealed an increased pressure gradient of $45 \mathrm{mmHg}$ across the tricuspid valve (Fig. 2C), consistent with pulmonary hypertension. Unexpectedly, from the subxiphoid view, a $13 \times 10 \mathrm{~mm}$ ostium secundum ASD with left-to-right shunt was detected (Fig. 2D and Supplementary material 3), and the pulmonary- 

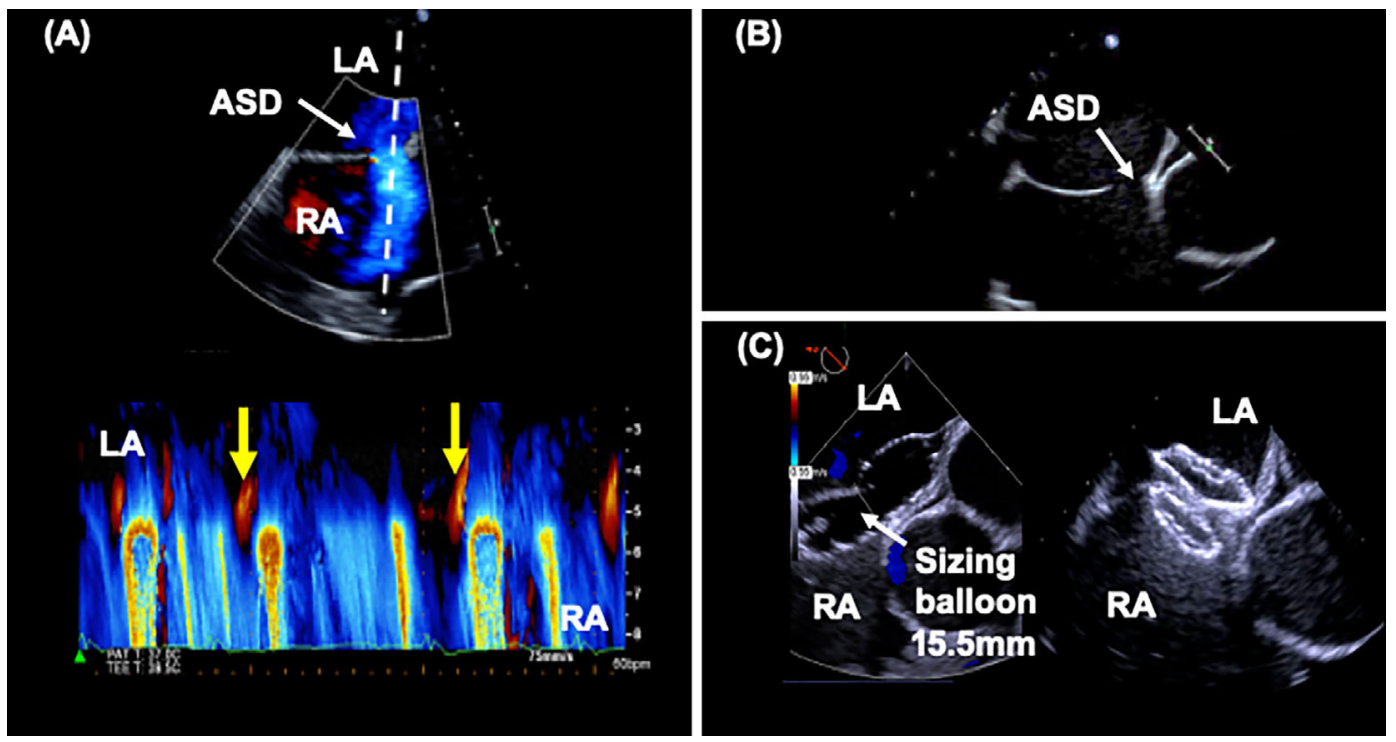

Figure 3. Transesophageal echocardiography of the 92-year-old male patient with an ostium secundum atrial septal defect (ASD). (A) A preoperative mid-esophageal sagittal view shows an ostium secundum ASD (white arrow). The M-mode echocardiogram clearly shows a dominant left-to-right shunt through the ASD with a small right-to-left shunt (yellow arrows) during early systole. (B) A preoperative mid-esophageal aortic valve short-axis view shows an ostium secundum ASD next to the sinus of Valsalva (white arrow). (C) Intraoperatively, the size of the ASD was measured using a sizing balloon (white arrow) and a Figulla Flex $\mathrm{II}^{\circledR}(21 \mathrm{~mm})$ occluder was successfully deployed. ASD: atrial septal defect

to-systemic-blood-flow ratio was 2.36. Transesophageal echocardiography confirmed a $13 \times 13 \mathrm{~mm}$ stium secundum ASD next to the sinus of Valsalva with a dominant left-toright, bidirectional shunt (Fig. 3A, B and Supplementary material 4). A cardiac catheter examination showed a pulmonary artery pressure of 34/8 $\mathrm{mmHg}$ (mean pressure 16 $\mathrm{mmHg}$ ), pulmonary vascular resistance of $84 \mathrm{dyn} \cdot \mathrm{s} \cdot \mathrm{cm}^{5}$, and a significant step-up of oxygen saturation from the superior vena cava $(75 \%)$ to the right ventricle $(91 \%)$.

Subsequently, the patient's heart failure proved to be refractory to the best-practice treatment with cardiotonic drugs [intravenous dobutamine $(3.5 \mu \mathrm{g} / \mathrm{kg} / \mathrm{min})]$, high-dose diuretics [furosemide (20 mg/day) and tolvaptan (15 mg/day)], and of warfarin ( $2 \mathrm{mg} /$ day). Further complicating the situation, the patient's renal function progressively deteriorated during this treatment, which negatively affected the hemodynamic control. In this case, the intractable heart failure was attributable to the combined hemodynamic disturbances, including an ostium secundum ASD with a large shunt burden, secondary tricuspid regurgitation, and atrial fibrillation with a slow ventricular response. The ASD was considered to play a central role in the condition of the patient. After a thorough discussion within the heart team, transcatheter closure was scheduled despite the fact that the patient was a nonagenarian with multiple comorbidities. On the 32nd hospital day, this procedure was performed under general anesthesia. Using an 18-mm balloon, a pre-procedural occlusion test was performed under right-sided catheter monitoring. This revealed the absence of a hemodynamic exacerbation. Because the measured defect size was $15.5 \mathrm{~mm}$ in diameter, to completely enclose the sinus of Valsalva, a Figulla Flex II $^{\circledR}$ occluder device (21 mm, Occlutech, Jena, Germany) was finally selected and successfully deployed without any complications (Fig. 3C). After device implantation, only clopidogrel $(75 \mathrm{mg} /$ day) was prescribed on top of warfarin to avoid the hemorrhagic complications.

Immediately after ASD closure, the patient's hemodynamics became controllable without the need for inotropic medication or intravenous diuretics, and his renal function gradually improved. The patient was discharged on the 9th day after ASD closure.

Surprisingly, the patient's heart returned to sinus rhythm, and not only the cardiomegaly but also the bilateral pleural effusion showed significant improvement at one month after ASD closure (Fig. 4). Moreover, despite the long-term volume overload, the right heart showed significant reverse remodeling (Supplementary material 5), and the cardiac output markedly improved from $2.8 \mathrm{~L} / \mathrm{min}$ to $4.4 \mathrm{~L} / \mathrm{min}$. At 10 months after ASD closure, his heart failure symptoms showed a marked improvement to NYHA functional class I. At present, he is caring for his sick wife in their own home.

\section{Discussion}

The clinical picture of an ASD in an elderly patient differs considerably from that in a younger patient. As a consequence of the long-standing left-to-right shunt, elderly patients suffer from a variety of complications, including pulmonary arterial hypertension, atrioventricular regurgitation, and various atrial arrhythmias, such as atrial fibrillation. As 

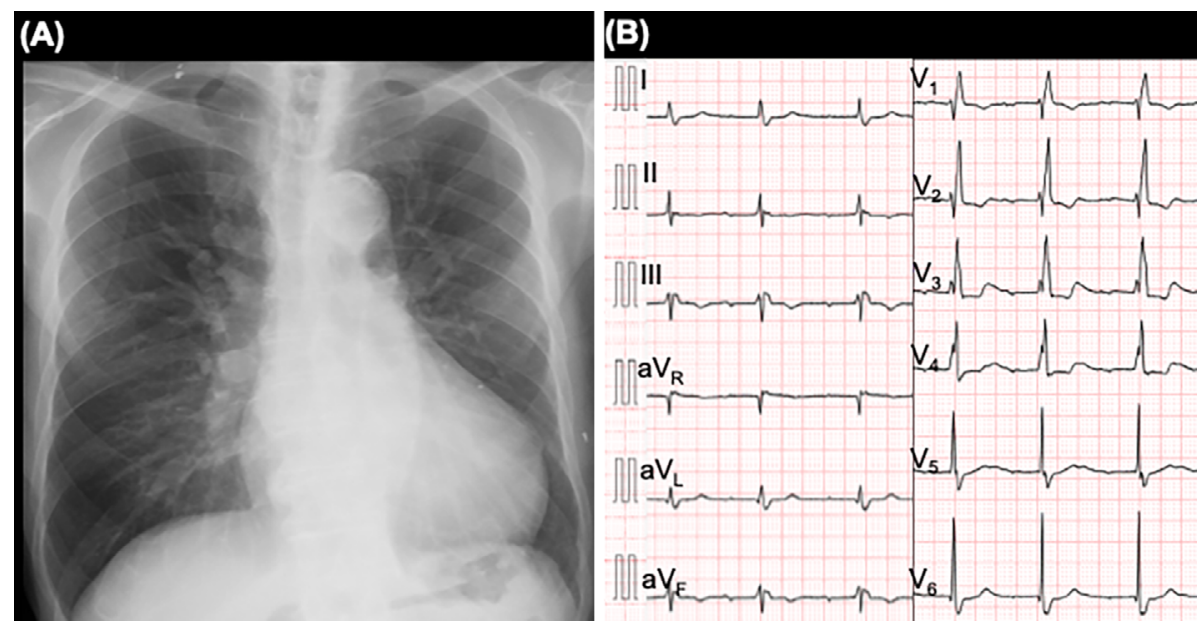

Figure 4. Radiography and electrocardiography of the 92-year-old male patient after transcatheter closure of the ostium secundum atrial septal defect. (A) A post-operative chest radiograph shows a significant reduction in the cardiothoracic ratio and the elimination of pleural effusion. (B) A postoperative electrocardiogram shows the restoration of sinus rhythm.

a result, their quality of life is decreased while morbidity and mortality are increased. Moreover, in some cases, the sequelae of long-standing ASD include severe right ventricular dysfunction, congestive liver disease with hypoalbuminemia, and renal dysfunction with the retention of sodium and water $(3,4)$. These complications make hemodynamic control even more difficult, eventually leading to intractable heart failure. Consequently, ASD closure has been withheld - or even omitted - in elderly patients because of the considerable surgical risk resulting due to various comorbidities (4).

On the other hand, transcatheter ASD closure was first described in humans in the 1970s (5) and has since been established as the standard treatment for ostium secundum ASD, representing a less invasive alternative to surgical closure (1). With the continuous development and broad implementation of new treatment strategies, the transcatheter technique has opened a new window of opportunity for elderly patients with ASD who had previously been excluded from curative treatment. In recent years, reports suggesting that transcatheter closure may even be beneficial for elderly patients with ASD have accumulated $(4,6,7)$. According to these reports, transcatheter ASD closure can be safely performed (6), and improves the exercise capacity and quality of life while reducing morbidity in these patients (6). It is noteworthy that reverse remodeling can be achieved after shunt closure (4), and the possibility of reduced mortality has also been reported in the elderly (7). It is possible that all of these favorable structural and hemodynamic improvements after ASD closure even showed beneficial effects on the heart rhythm in our case. However, even though transcatheter ASD closure is increasingly considered to be effective in elderly patients, to date, there has been no information on the safety and clinical benefits in "super-elderly" patients, such as the 92-year-old patient in the present case.

We presented the case of a nonagenarian with intractable heart failure who was successfully treated by transcatheter ASD closure. We could confirm right ventricular reverse remodeling and an increase in cardiac output after the closure. Moreover, we observed secondary effects, such as restoration of sinus rhythm and improvement of the renal function. All of these changes favorably influenced the hemodynamics and contributed to the improved quality of life and exercise tolerance in our patient after the procedure. Thus, even for the super-elderly, transcatheter ASD closure can be beneficial and should be considered as a treatment option.

\section{Conclusions}

To the best of our knowledge, this is the oldest patient with ASD in the world to be successfully treated with transcatheter shunt closure. The main message of this case is that transcatheter ASD closure has considerable potential for the effective treatment of super-elderly patients with ASD because of its minimal invasiveness.

The authors state that they have no Conflict of Interest (COI).

\section{References}

1. Baumgartner H, Bonhoeffer P, De Groot NM, et al. ESC Guidelines for the management of grown-up congenital heart disease (new version 2010). Eur Heart J 31: 2915-2957, 2010.

2. Van De Bruaene A, La Gerche A, Prior DL, Voigt JU, Delcroix M, Budts W. Pulmonary vascular resistance as assessed by bicycle stress echocardiography in patients with atrial septal defect type secundum. Circ Cardiovasc Imaging 4: 237-245, 2011.

3. Nakagawa K, Akagi T, Taniguchi M, et al. Transcatheter closure of atrial septal defect in a geriatric population. Catheter Cardiovasc Interv 80: 84-90, 2012.

4. Khan AA, Tan JL, Li W, et al. The impact of transcatheter atrial septal defect closure in the older population: a prospective study. JACC Cardiovasc Interv 3: 276-281, 2010.

5. King TD, Thompson SL, Steiner C, et al. Secundum atrial septal defect nonoperative closure during cardiac catheterization. JAMA 
235: 2506-2509, 1976.

6. Humenberger M, Rosenhek R, Gabriel H, et al. Benefit of atrial septal defect closure in adults: impact of age. Eur Heart J 32: 553560, 2011.

7. Takaya Y, Akagi T, Kijima Y, Nakagawa K, Sano S, Ito H. Longterm outcome after transcatheter closure of atrial septal defect in older patients: impact of age at procedure. JACC Cardiovasc In- terv 8: 600-606, 2015 .

The Internal Medicine is an Open Access journal distributed under the Creative Commons Attribution-NonCommercial-NoDerivatives 4.0 International License. To view the details of this license, please visit (https://creativecommons.org/licenses/ by-nc-nd/4.0/).

(C) 2020 The Japanese Society of Internal Medicine Intern Med 59: 1059-1063, 2020 